\title{
Separation of Colloidal Particle Using Elastic-Gel-Packed Column
}

\author{
Hidetaka KAWAKITA*, Kaori YoKOYAMA, Shohei EsAKI, Shintaro MorISADA, and Keisuke OHTO \\ Faculty of Science and Engineering, Saga University, 1-Honjo, Saga 840-8502, Japan
}

\begin{abstract}
Polymerized spherical gel $(46 \mu \mathrm{m})$ was packed in a column to permeate silica particle suspension for the separation due to their differences of sizes. The elastic gel in the bottom domain of column deformed more because the pressure of the fluid flow suppressed the packed gel layer, that is, the formed gaps among the gels at the bottom domain had the smaller 'pore', resulting in filtering the smaller-size of the particles at the bottom of the column. The larger the size of silica particle, the upper the filtered domain in the column. To elute the filtered silica particle, the gel layer was compacted and restored by on-off of the fluid flow, to open the gaps in the gel layer repeatedly, demonstrating that the elution percentage of silica particle was gradually increased by the repeated on-off permeation of water.
\end{abstract}

\section{Introduction}

Several methods to separate various colloidal particles such as ceramics, crystal, cells and microorganisms, have been proposed. Microfiltration by the membrane (Zhang, et al., 2006), hydrodynamic chromatography by fluid flow through the circular column (Miyagawa et al., 2016), and field-flow fractionation by the fluid flow along with the tangential flow (Moon and Gidding, 1993) were studied. Colloidal particles have the various sizes and morphology that the sophisticated and easy separation methods have been required.

Spherical gel particles hold water molecules in a hydrated polymer matrix. Gel packed in column has been used for gel permeation chromatography and the support of enzyme immobilization. Through the deformable gel packed column, as water was permeated, gel can be deformed to ellipsoidal and polyhedral shapes under the action of an external mechanical force of fluid flow ( $\mathrm{Lu}$ et al., 2001; Ueyama and Furusaki, 1985). Gel deformation in a chromatographic column is typically studied under a high-speed fluid flow mathematically by Östergren and Trägrådh (1999). The lower domain in the gel feels more forces, resulting in the more deformation of the gel, forming the narrower pore among the gels.

So far, colloidal particle separation using the dynamic elastic behavior of the gel layer packed in column has been proposed (Takaoka et al., 2017; Takaoka et al., 2018; Takaoka et al., 2019). The elastic gel feels more force by the fluid flow, as a result, forming the compacted structure. When the colloidal suspension was permeated to the gel layer, the colloidal particle at the smaller size is filtered by the gaps of the gels. At the lower domain in the column, the colloidal particle at the more smaller size was filtered. Silica particle produced by dry process and

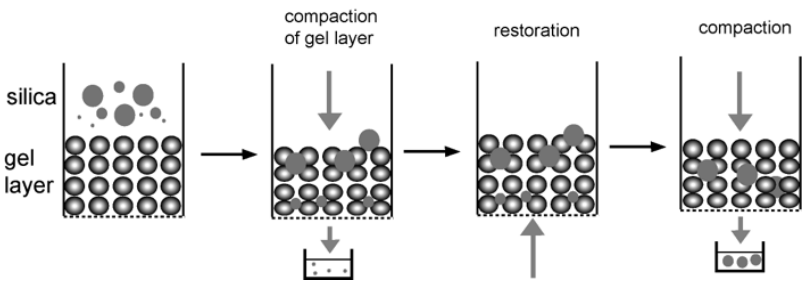

Figure 1. Proposed elution method of the filtered particles by on-off permeation of water through elastic gel layer

graphene oxide was separated by the elastic gel layer due to the difference of sizes and morphology. However, the recovery methods of the filtered particles remained among the gel layer has not yet proposed.

In this study, the elution method of the filtered colloidal particles has proposed: spherical polymerized gel was packed in the column and the suspension of silica particle was injected to the top of the packed-gel layer. Water was permeated to filter the silica particle at each domain in the gel layer. Repeated on-off flow of the water makes the gel layer compacted and restored, eluting the filtered particle, by opening the gaps among the gel gradually, as shown in Figure 1. The eluted silica particle was observed and the size distribution was quantitatively determined.

\section{Experiment}

\subsection{Materials}

Monomer, crosslinker, and initiator used were $\mathrm{N}, \mathrm{N}$ dimethylacrylamide $\quad(049-19185), \quad N, \quad N^{\prime}$-methylene bisacrylamide (M0506), and ammonium persulfate

\footnotetext{
Corresponding author: kawakita@cc.saga-u.ac.jp
} 
(018-03282) obtained from Wako Pure Chemical Industries, Japan. Span 80 (S0060) and Tween 80 (T2533) were obtained from Tokyo Chemical Industry Co., Japan. Glass beads (105-125 $\mu \mathrm{m}, \mathrm{BZ}-01)$ was purchased from AS-ONE, Japan. Silica particles of $0.12 \mu \mathrm{m}$ (measured size: $170 \mathrm{~nm}$, polydispersity index (PI) 0.029), $1.0 \mu \mathrm{m}$ (measured size $1.2 \mu \mathrm{m}$, PI 0.0080), and $10 \mu \mathrm{m}(7.5 \mu \mathrm{m}$, PI 1.7) were from JGC, C\&C, and Corefront Corporation, respectively. Industrial silica particle was donated by Nitchitsu Co., Ltd., Japan. The small column (Bio-rad corporation) used had the inner diameter of $0.5 \mathrm{~cm}$ and height of $10 \mathrm{~cm}$, and the large one had that of $1.25 \mathrm{~cm}$ and that of $10 \mathrm{~cm}$. Other chemicals were of analytical grade or higher.

\subsection{Preparation of spherical gel}

A water phase that was comprised of monomer, crosslinker, and initiator was dropped into an organic phase of hexane to obtain spherical gel particles via suspension polymerization (Yao, et al., 2013). Span 80 and Tween 80 were dissolved in hexane in a three-neck flask (500 mL). $N, N$-Dimethylacrylamide (0.086 mol), $N$, $N$ '-methylenebisacrylamide $(0.0095 \mathrm{~mol})$, and water setting were mixed, after which ammonium persulfate was added and dissolved in the mixture. The molar ratio of monomer and crosslinker was set at $9: 1$. The water phase was dropped into the organic phase using a dropping funnel to start the polymerization at $348 \mathrm{~K}$ with stirring at $360 \mathrm{rpm}$ for $1 \mathrm{~h}$. The width of stirring blade was $8 \mathrm{~cm}$. The obtained gel particles were recovered by centrifugation at $6000 \mathrm{rpm}$ for $15 \mathrm{~min}$. The gel particles were washed with ethanol and water three times. The resulting gel particles were stored in water at $10.0 \mathrm{wt} \%$. The gel sample was analyzed by optical microscopy (VH$\mathrm{S} 5$, Keyence) to determine the size distribution from more than 200 particles.

\subsection{Permeation of silica particle suspension through the gel layer}

To prepare the gel layer in column, firstly glass bead $(0.3$ g) was packed and then gel suspension $(1.8 \mathrm{~mL}, 10 \mathrm{wt} \%)$ was added to the small column. Suspension of silica particles having a diameter of $0.12 \mu \mathrm{m}, 1.0 \mu \mathrm{m}$, and 10 $\mu \mathrm{m}$ was added on the top of the gel layer and water was permeated through the column using syringe pump. Flow rate of water was set at $15 \mathrm{~mL} / \mathrm{h}$. Injected concentration and volume of suspension of silica particle on the gel layer was set at $1.0 \mathrm{~g} / \mathrm{L}$ and $0.6 \mathrm{~mL}$, respectively. During the permeation, the pressure drop, the weight of effluent from the column as well as the concentration of silica particle in the effluent was determined continuously. Concentration of silica particle was determined at $600 \mathrm{~nm}$ by UV-VIS.

For separation of industrial silica particle, the gel suspension (35 mL, $10 \mathrm{wt} \%$ ) was added to the large column. Instead of the suspension of silica particle, industrial silica suspension $(10 \mathrm{~g} / \mathrm{L}, 1.5 \mathrm{~mL})$ was permeated. The gel layer was compacted and restorated
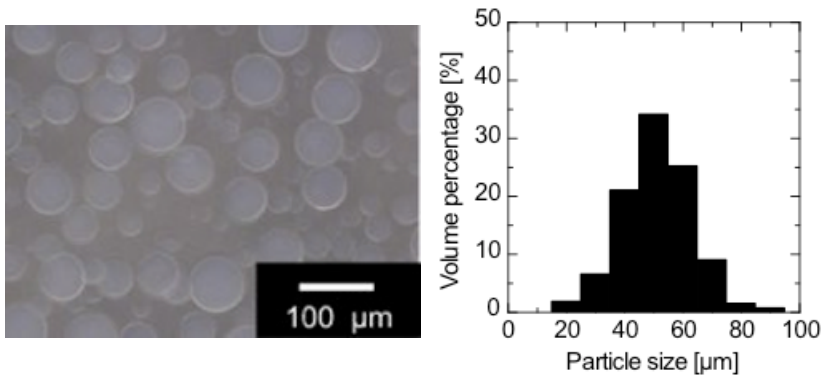

Figure 2. Images and size distribution of prepared spherical gel
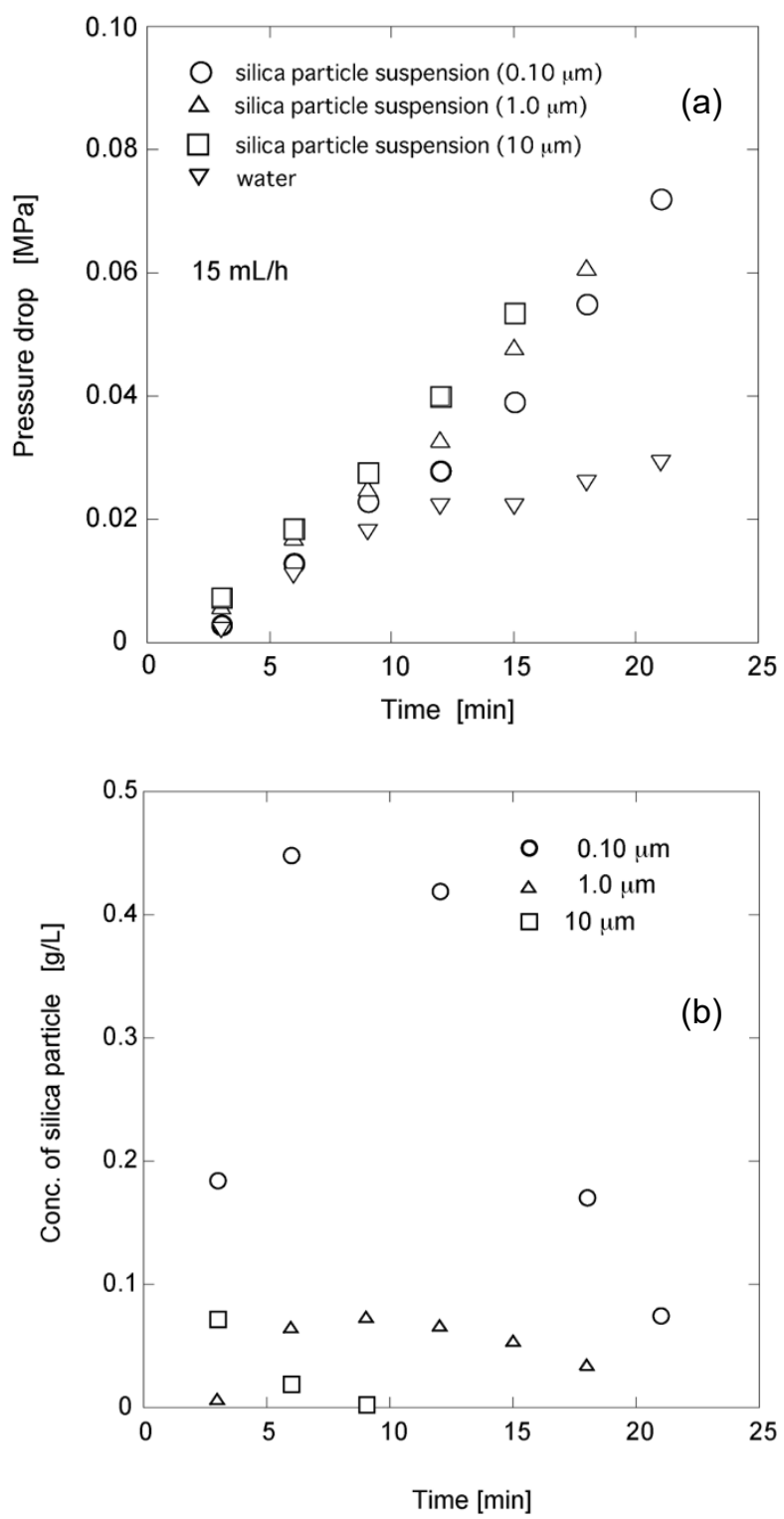

Figure 3. Individual permeation of silica particle suspension through the gel layer. (a) pressure drop, and (b) concentration of silica particle in the effluent.

by the on-off permeation of water by changing the flow rates from $375 \mathrm{~mL} / \mathrm{h}$ to $75 \mathrm{~mL} / \mathrm{h}$. In change of the flow rate, the gel layer was left for one-hour interval. During the on-off, silica particles filtered among the gels were eluted gradually, and the pressure drop, height of the gel layer and concentration of silica particle were determined. 

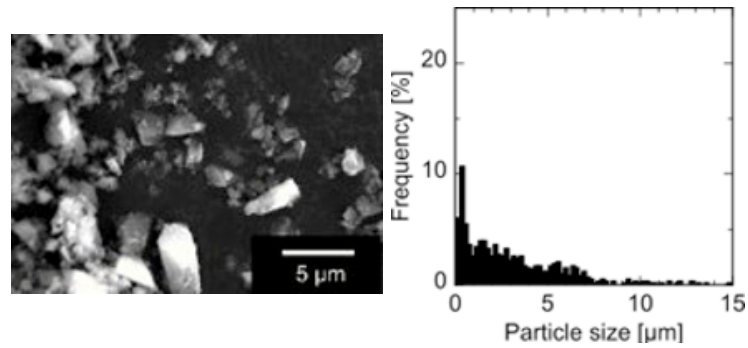

Figure 4. Images and size distribution of industrial silica particle

\section{Results and Discussion}

\subsection{Individual permeation of silica suspension through gel layer}

Water-in-oil suspension polymerization is a technique for producing large amounts of gel particles. Typically, a soluble monomer and initiator are dissolved in a water solution, and then, the solution is dropped in oil. The prepared spherical gel was shown in Figure 2. The mean size of the gel was $46 \mu \mathrm{m}$. This gel has the threedimensional network and would has the elasticity due to the pressure by the fluid flow.

The spherical gel was packed in a column and suspension of silica particles $(0.12 \mu \mathrm{m}, 1.0 \mu \mathrm{m}$, and 10 $\mu \mathrm{m}$ ) was individually injected on the gel layer, permeating water through the column. The time course curves of pressure drop and concentration of silica particle in the effluent are shown in Figure 3 (a). Compared with the water permeation, due to the filtration of silica particle by the gel layer, the pressure drop was larger. As shown in Figure 3 (b), the concentration of silica particle in the effluent increased in the case of $0.12 \mu \mathrm{m}$, because the silica particle with the smaller size easily passed through the gel layer, while the larger were filtered among the gaps of the deformed gels. Percentages of filtered silica particles for $0.12 \mu \mathrm{m}, 1.0 \mu \mathrm{m}$, and $10 \mu \mathrm{m}$ were $57 \%, 94 \%$, and $95 \%$, respectively, demonstrating that particles in the range between $0.12 \mu \mathrm{m}$ and $1.0 \mu \mathrm{m}$ was suitable for separation using this gel layer.

\subsection{Recovery of filtered industrial silica particle by on-off of gel layer}

The silica particles at the smaller size was eluted through the gel layer, while the larger one was filtered by the deformation of gel layer. Repeated compaction and restoration of the gel layer could elute the filtered particles, as proposed in Figure 1. The images and size distribution of industrial silica particles used was shown in Figure 4. Mean diameter of the silica particle was $3.2 \mu \mathrm{m}$, but the silica had the wide distribution to more than $1 \mu \mathrm{m}$. After the injection of the suspension of the industrial silica particle on the gel layer, water was permeated at the flow rate of $375,325,225$, and $75 \mathrm{~mL} / \mathrm{h}$ to be changed. Pressure drop, height of the gel layer, and concentration of silica particle are shown in Figure 5. At the high flow rate (375
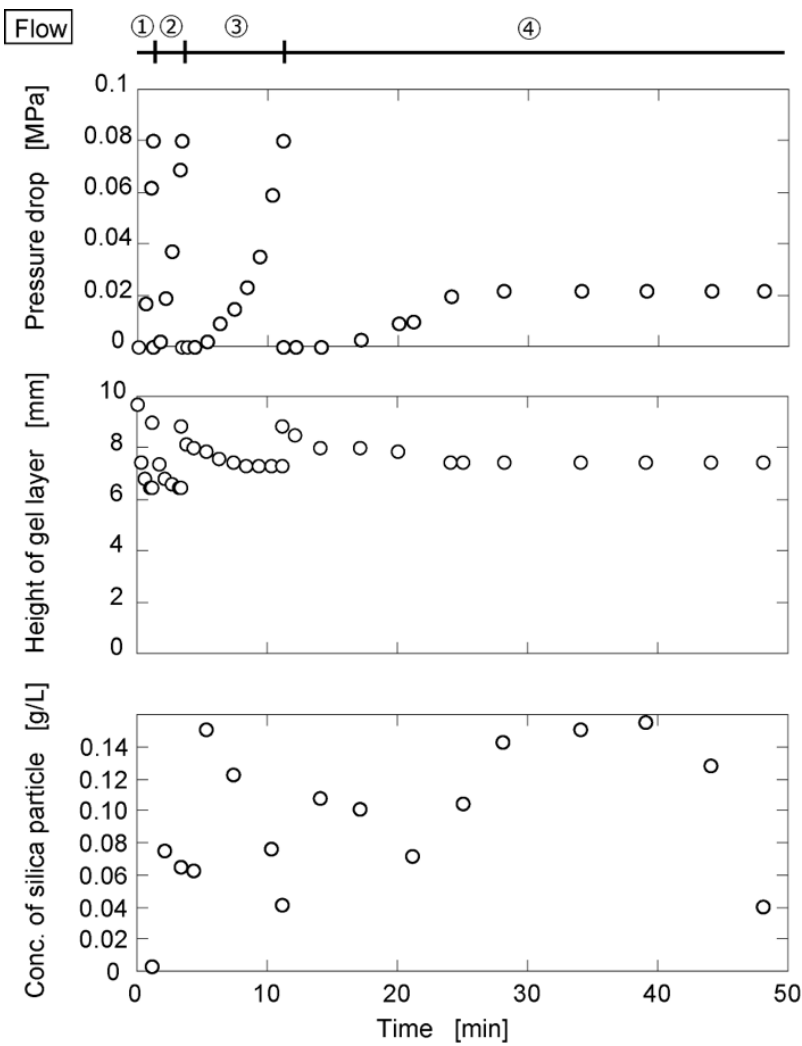

Figure 5. Repeated on-off permeation of gel layer for elution of filtered silica particle. Flow rate of water ; (1) $375 \mathrm{~mL} / \mathrm{h}$, (2) $325 \mathrm{~mL} / \mathrm{h}$, (3) $225 \mathrm{~mL} / \mathrm{h}$, and (4) $75 \mathrm{~mL} / \mathrm{h}$
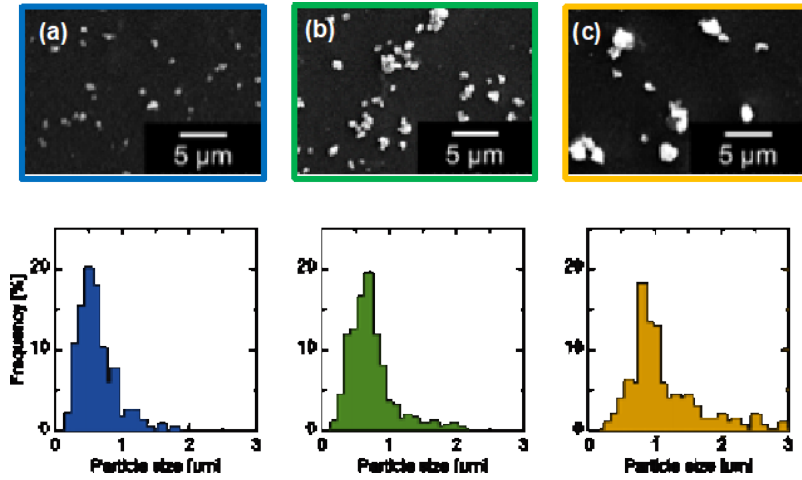

Figure 6. SEM images and size distribution of the eluted silica particles at (a) $325 \mathrm{~mL} / \mathrm{h}$, (b) $225 \mathrm{~mL} / \mathrm{h}$, and (c) $75 \mathrm{~mL} / \mathrm{h}$

$\mathrm{mL} / \mathrm{h}$ ), the pressure drop increased exponentially due to the deformation of the gel layer. In the permeation of water, the height of the gel layer was reduced by the pressure of the flowing fluid. The concentration of silica particles flowed from the column was less because the compaction of the gel layer occurred rapidly to close the path for silica particle in gel layer.

After the compaction of the gel layer, the flow rate of water was changed from $375 \mathrm{~mL} / \mathrm{h}$ to $325 \mathrm{~mL} / \mathrm{h}$, again the pressure drop increased exponentially, and the gel layer was compacted, and concentration of silica particle in the effluent was increased. Compared with the higher flow rate $(375 \mathrm{~mL} / \mathrm{h})$, the eluted silica particles were increased because compaction speed of the gel layer was slower and 
filtered silica particle could be eluted by convection of flowing water. To change the flow rate to $375,325,225$, and $75 \mathrm{~mL} / \mathrm{h}$, the eluted percentage increased $0,3.6 \%$, $9.2 \%$, and $27 \%$, respectively, demonstrating that compaction and restoration of the gel layer by repeated on-off flow of the fluid make the filtered silica particles eluted. The eluted particle images of SEM are shown in Figure 6 along with the size distribution. This indicated that the smaller silica particles were gradually eluted at the changed lower flow rate of water due to the compaction of restoration of the gel layer. The obtained silica particles had the mean sizes of $0.61 \mu \mathrm{m}, 0.72 \mu \mathrm{m}$ and $1.09 \mu \mathrm{m}$ at the flow rate of 325,225 , and $75 \mathrm{~mL} / \mathrm{h}$, respectively. The hydrodynamic on-off of the gel layer has the possibility of elution of filtered silica particle, which will enhance the potential of application of deformable gel layer for separation of several particles.

\section{Conclusions}

The spherical gel was packed in column and the silica particle suspension was injected on the elastic gel layer, permeating water through the column for separation of silica particle by pore distribution formed by gaps among the gels in flow direction. The filtered silica particle was eluted by the restoration of the gel layer to control the flow rate of water because the gaps among the gel layer were opened, to easily move the particles by convection of water. The repeated compaction and restoration of the gel layer enables to elute the filtered silica particle gradually. The elastic gel layer provides the separation of colloidal particle by the gel layer along with the potential of eluting the filtered silica particles.

\section{Acknowledgements}

This research is partially supported by Adaptable and Seamless Technology transfer Program through Target-driven R\&D (ASTEP) from Japan Science and Technology Agency (JST). SEM used belongs to Analytical Research Center for Experimental Sciences, Saga University.

\section{References}

Lu, W.-M., K.-L. Tung, S.-M. Hung, J.-S. Shiau, and K.J., Hwang; "Constant Pressure Filtration of MonoDispersed Deformable Particle Slurry," Sep. Sci. Technol., 36, 2355-2383 (2001)

Miyagawa, Y., S. Morisada, K. Ohto, and H. Kawakita; "Hydrodynamic Chromatography Using Flow of a Highly Concentrated Dextran Solution through a Coiled Tube," Carbohydr. Polym., 146, 109-114 (2016)

Moon, M. H. and J. C. Gidding; "Rapid Separation and Measurement of Particle Distribution of Starch Granules by Sedimentation/Steric Field-Flow Fractionation," $J$. Food Sci., 58, 1166-1171 (1993)
Östergren, K. C. E. and C. Trägrådh; "Modelling and Analysis of Axial Flow through and Compression of a Non-Rigid Chromatographic Bed," Chem. Eng. J., 72, 153-161 (1999)

Takaoka, Y., S. Morisada, K. Ohto, and H. Kawakita; "Filtration of Colloidal Particles using Compacted-Gel Media Packed in a Column," J. Chem. Eng. Japan., 50, 815-820 (2017)

Takaoka, Y., M. Miyoshi, K. Sakaguchi, S. Morisada, K. Ohto, and H. Kawakita; "Recovery of Filtered Graphene Oxide Residue Using Elastic Gel Packed in a Column by Cross Flow," Processes, 6, 43 (2018)

Takaoka Y., S. Esaki, K. Sakaguchi, T. Fujisawa, M. Unno, S. Morisada, K. Ohto, and H. Kawakita; "SizeDependent Separation of Graphene Oxide by Deformation of Packed-Gel in a Chromatographic Column," Sep. Sci. Technol., 55, 1356-1363 (2019)

Ueyama, K. and S. Furusaki; "A Theoretical Study on the Effect of Compaction on the Effectiveness Factor of Gel Particles Containing Immobilized Enzymes," Chem. Eng. Commun., 36, 299-316 (1985)

Yao, C., G. Lei, X. Gao, and L. Li; "Controllable Preparation, Rheology, and Plugging Property of MicronGrade Polyacrylamide Microspheres as a Novel Profile Control and Flooding Agent," J. Appl. Polym. Sci., 130, 1124-1130 (2013)

Zhang, Y. P., A. G. Fane, and A. W. K. Law; "Critical Flux and Particle Deposition of Bidisperse Suspensions during Crossflow Microfiltration," J. Membr. Sci., 282, 189-197 (2006) 